\title{
CELL-LIKE SPACES
}

\author{
R. C. LACHER ${ }^{1}$
}

In this note we give a characterization of those compacta which can be embedded in manifolds as cellular sets (the cell-like spaces). There are three conditions equivalent to cell likeness for a finitedimensional compactum $X$. One of these is that $X$ have the Cechhomotopy-type of a point, as defined by Borsuk in [1]. Another is a technical condition which implies that McMillan's cellularity criterion [5] holds, not just for compact absolute retracts but for arbitrary cell-like spaces. It follows that most of the theorems of [5] hold for cell-like subsets of manifolds.

Definition. A subset $X$ of the $n$-manifold $N$ is said to be cellular in $N$ if there exists a sequence $Q_{1}, Q_{2}, \cdots$ of topological $n$-cells in $N$, with $X \subset Q_{i+1} \subset$ Int $Q_{i}$ for each $i$, such that $X=\bigcap_{i=1}^{\infty} Q_{i}$.

Clearly cellularity is a property of the embedding of $X$ in $N$. However, there is the corresponding intrinsic topological property, as follows:

Definition. A space $X$ is cell-like if there is an embedding $f$ of $X$ into some manifold $N$ such that $f(X)$ is cellular in $N$.

Cellularity was first defined by Brown in [3]. Since then, the idea has been important in the study of manifolds, and hence the problem of recognizing cellular sets has also been important. By far the best recognition criterion was given by McMillan in [5]. There he proved that, if $X$ is a compact absolute retract in the interior of the combinatorial $n$-manifold $N, n \geqq 5$, then $X$ is cellular in $N$ provided that the inclusion $X \subset N$ has property $\left(^{*}\right)$ below.

Property (*). An embedding $f: X \rightarrow Y$ is said to have property $\left({ }^{*}\right)$ if, for any open set $U$ of $Y$ containing $f(X)$, there is an open set $V$ of $Y$, with $f(X) \subset V \subset U$, such that any loop in $V-f(X)$ is contractible in $U-f(X)$.

McMillan notes, however, that the only property of compact absolute retracts which is needed for his proof is that any embedding of a compact absolute retract into a manifold has property (**) below.

Property (**). An embedding $f: X \rightarrow Y$ is said to have property (**) if, for any open set $U$ of $Y$ containing $f(X)$, there is an

Received by the editors January 20, 1968.

1 Supported by the National Science Foundation. 
open set $V$ of $Y$, with $f(X) \subset V \subset U$, such that the inclusion $V \subset U$ is null-homotopic (in $U$ ).

Thus, McMillan actually proves that the compactum $X$ in the interior of the combinatorial $n$-manifold $N, n \geqq 5$, is cellular in $N$ if and only if the inclusion $X \subset N$ has properties $\left({ }^{*}\right)$ and $\left({ }^{* *}\right)$. (See the second paragraph of $\$ 1$ of $[5]$.) We will show that any embedding of a cell-like space into an ANR has property $\left.{ }^{* *}\right)$. In fact, our main result can now be stated.

[Henceforth, for simplicity, we will assume that all manifolds are manifolds-without-boundary and that all ANR's are retracts of neighborhoods of euclidean spaces. We do not assume that manifolds or ANR's are compact.]

TheOREM. Let $X$ be a finite-dimensional compact metric space. Then the following conditions are equivalent:

(a) $X$ is cell-like.

(b) $X$ has the Cech-homotopy-type of a point, as in [1].

(c) There exists an $A N R, N$, and an embedding $f: X \rightarrow N$ which has property $\left({ }^{* *}\right)$.

(d) For any $A N R, N$, any embedding $f: X \rightarrow N$ has property (**).

Since any manifold is an ANR [4], we have the following

Corollary 1. Let $M$ be $R^{3}, S^{3}$, or a combinatorial manifold of dimension $\geqq 5$, and let $X$ be a cell-like subset of $M$. Then $X$ is cellular in $M$ if and only if the inclusion $X \subset M$ has property (*).

Corollary 2. Theorems $1,1^{\prime}, 2,2^{\prime}, 5,7$, and 8 of [5] remain valid if the assumption that $X$ be an absolute retract is weakened to the assumption that $X$ be cell-like.

The proof of the Theorem will be according to the following plan: $(a) \Rightarrow(b) \Rightarrow(c) \Rightarrow(a)$, and $(a) \Rightarrow(d)$. Since it is obvious that $(d) \Rightarrow(c)$, the theorem will follow from these four implications.

Proof that $(a) \Rightarrow(b)$. Let $X$ be a cell-like space. We may as well assume that $X$ is a cellular subset of $R^{n}$ for some $n$. [ $R^{n}$ is euclidean $n$-space.] Let $Q_{1}, Q_{2}, \cdots$ be a sequence of $n$-cells in $R^{n}$, with $Q_{i+1} \subset$ Int $Q_{i}$, whose intersection is $X$. By pulling in slightly on each $Q_{i}$, we may assume that $\mathrm{Bd} Q_{i}$ is collared in $R^{n}-\operatorname{Int} Q_{i}$ for each $i$. (See [3].) For each $i$, let $R_{i}$ be the closed ball in Hilbert space $H$, with center zero, of diameter $1 / i$. Finally, let $B_{i}=Q_{i} \times R_{i}$. Under the identification $R^{n} \times H=H, B_{i}$ is a sequence of closed sets in $H$ whose intersection is $X \times 0=X$. We define $B_{i}^{\circ}$ to be Int $Q_{i} \times R_{l}^{\circ}$, where $R_{l}^{\circ}$ is the open ball of radius $1 / i$. Note that $B_{i}^{\circ}$ is open in $H$ and the closure of $B_{i}^{\circ}$ is $B_{i}$. 
Let $x_{0}$ be a point of $X$. We want to define fundamental sequences $\left\{f_{i}\right\}: X \rightarrow\left\{x_{0}\right\}$ and $\left\{g_{i}\right\}:\left\{x_{0}\right\} \rightarrow X$ whose compositions are equivalent to the appropriate identity classes. In order to define $\left\{f_{i}\right\}$ we need some neighborhoods of $x_{0}$. For each $i$, let $q_{i}$ be the closed ball in $R^{n}$ with center $x_{0}$ and radius $1 / i$. Define $b_{i}$ to be $q_{i} \times R_{i}$. Now, for each $i$, define a map $f_{i}: R^{n} \times H \rightarrow R^{n} \times H$ by

$$
f_{i}=h_{i} \times \text { (identity) }
$$

where $h_{i}$ is a homeomorphism of $R^{n}$ taking $Q_{i}$ to $q_{i}$ and $x_{0}$ to $x_{0}$. (Such $h_{i}$ always exist. See [3].)

Clearly, $f_{i}$ is a homeomorphism of $H$ onto itself taking $B_{i}$ onto $b_{i}$. Let $g_{i}=f_{i}^{-1}$. It is easily checked that $\left\{f_{i}\right\}: X \rightarrow\left\{x_{0}\right\}$ and $\left\{g_{i}\right\}:\left\{x_{0}\right\}$ $\rightarrow X$ are fundamental classes of maps (in the sense of [1]). It is obvious that $\left\{g_{i}\right\}\left\{f_{i}\right\}=\left\{\operatorname{id}_{x}\right\}$ and $\left\{f_{i}\right\}\left\{g_{i}\right\}=\left\{\operatorname{id}_{\left\{x_{0}\right\}}\right\}$.

Proof that $(b) \Rightarrow(c)$. Let $X$ have the Cech-homotopy-type of a point. We will in fact show that any embedding of $X$ into $R^{n}$ has property (**). Assume that $X \subset R^{n}=R^{n} \times 0 \subset H$.

Let $\left\{f_{i}\right\}: X \rightarrow\left\{x_{0}\right\}$ be a fundamental sequence. According to [1], the sequence $\left\{f_{i}\right\}$ is generated by a map $f: X \rightarrow\left\{x_{0}\right\}$, since $\left\{x_{0}\right\}$ is an absolute retract. Hence, we may assume that each $f_{i}$ is the map $H \rightarrow\left\{x_{0}\right\}$.

Let $\left\{g_{i}\right\}:\left\{x_{0}\right\} \rightarrow X$ be a fundamental sequence such that $\left\{f_{i}\right\}\left\{g_{i}\right\}$ $\cong\left\{\operatorname{id}_{\left\{x_{0}\right\}}\right\}$ and $\left\{g_{i}\right\}\left\{f_{i}\right\} \cong\left\{\operatorname{id}_{x}\right\}$. Let $U$ be an open set in $H$ containing $X$. Now, choose $V$ open in $H$ such that $X \subset V \subset U$ and $\left.g_{i} f_{i}\right|_{V} \cong \mathrm{id}_{V}$ in $U$ for almost all $i$. Then, since $f_{i}$ is the constant map, $g_{i} f_{i}$ is constant, and hence $\mathrm{id}_{V} \cong$ constant in $U$. Thus we have proved that the inclusion $X \subset H$ has property $\left({ }^{* *}\right)$. To see that the inclusion $X \subset R^{n}$ has property $\left({ }^{* *}\right)$, let $U_{1}$ be an open set in $R^{n}$ containing $X$, let $U_{2}$ be an open ball in $H$, and let $U=U_{1} \times U_{2}$. Then, by the above, there is an open set $V$ of $H$, with $X \subset V$, such that the inclusion $V \subset U$ is null-homotopic. Let $V_{1}=V \cap R^{n} \times 0$. Since $U$ deforms onto $U_{1}$, it is clear that the inclusion $V_{1} \subset U_{1}$ is null-homotopic. Hence the inclusion $X \subset R^{n}$ has property $\left({ }^{* *}\right)$.

Proof That (c) $\Rightarrow$ (a). Let $X \subset N$ have property $\left({ }^{* *}\right)$, where $N$ is an ANR. It follows immediately that some embedding of $X$ in $R^{n}$ has property $\left(^{* *}\right)$, since any embedding of $N$ into $R^{n}$ has a similar property.

Hence, we assume that $X \subset R^{n}$ has property $\left({ }^{* *}\right)$ for some $n \geqq 2$. We will show that $X \times 0$ is cellular in $R^{n+3}=R^{n} \times R^{3}$. By McMillan's theorem, we need only show that the inclusion $X \subset R^{n+3}$ has properties $\left({ }^{*}\right)$ and $\left({ }^{* *}\right)$. Condition $\left({ }^{* *}\right)$ holds by the above paragraph. 
To verify property $\left({ }^{*}\right)$, let $U_{0}$ and $V_{0}$ be open sets in $R^{n}$ with $X \subset V_{0}$ $\subset U_{0}$, and assume that the inclusion $V_{0} \subset U_{0}$ is null-homotopic. Let $B$ be a small 3-ball in $R^{3}$, centered at the origin. Define $U=U_{0}$ $\times$ Int $B$ and $V=V_{0} \times \operatorname{Int} B$. Let $f: S^{1} \rightarrow(V-X \times 0)$ be a loop in $V-(X \times 0)$. By a general position argument, we may assume that $f\left(S^{1}\right)$ does not intersect $V_{0} \times 0$. That is, $f$ maps $S^{1}$ into $V-V_{0} \times 0$. Let $S$ be the boundary of the ball in $R^{3}$ with one-half the radius of $B$. Then there is a deformation retraction of $U-U_{0} \times 0$ onto $U_{0} \times S$ which restricts to one of $V-V_{0} \times 0$ onto $V_{0} \times S$. Thus, $f$ is homotopic (in $V-X$ ) to a loop in $V_{0} \times S$. Clearly, then, $f$ is contractible in $U_{0} \times S \subset U-X$, since $S$ is simply connected. Since $X$ has arbitrarily small neighborhoods in $R^{n+3}$ of the form $U_{0} \times \operatorname{Int} B$, the proof is complete.

Proof that (a) $\Rightarrow(\mathrm{d})$. Let $X$ be a cell-like subset of $N$, an ANR. Let $f: X \rightarrow Q_{1}$ be an embedding of $X$ into the $n$-cell $Q_{1}$, where $f(X)$ is the intersection of $Q_{1}, Q_{2}, \cdots$, and where each $Q_{i}$ is an $n$-cell with $Q_{i+1} \subset$ Int $Q_{i}$. By Tietze's Extension Theorem [2], there is a map $F: N \rightarrow Q_{1}$ which extends $f$. Moreover, by the neighborhood extension property of ANR's [2], there is an integer $j$ and a map $G: Q_{j} \rightarrow N$ which extends $f^{-1}$. Thus we have the following commutative diagram of maps:

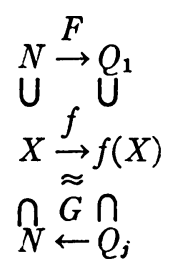

Let $U$ be a neighborhood of $X$ in $N$. Then $G^{-1}(U)$ is open, so $Q_{k} \subset G^{-1}(U)$ for some $k>j$. Let $V^{\prime}=F^{-1}\left(\right.$ Int $\left.Q_{k}\right) \cap U$. Notice that $\left.G F\right|_{V^{\prime}}$ is null homotopic in $U$, since $G\left(Q_{k}\right) \subset U$ and $Q_{k}$ is contractible. Therefore, to complete the proof, we need to find an open set $V$ in $N$, with $X \subset V \subset V^{\prime}$, such that the inclusion $V \subset U$ is homotopic to $\left.G F\right|_{V}$ in $U$.

Let $H$ be the map of $Y=X \times I \cup V^{\prime} \times 0 \cup V^{\prime} \times 1$ into $U$ which is the identity on $X \times I \cup V^{\prime} \times 0$ and is $G F$ on $V^{\prime} \times 1$. Again, using the neighborhood extension property, we can extend $H$ to a map of $W$ into $U$, where $W$ is an open set of $V^{\prime} \times I$ containing $Y$. Let $V$ be an open set of $N$, containing $X$, such that $V \times I \subset W$. Then $H \mid V \times I$ is a homotopy between the inclusion $V \subset U$ and $\left.G F\right|_{v}$, so the proof is complete. 


\section{REFERENCES}

1. K. Borsuk, Concerning homotopy properties of compact subsets of Hilbert space, Fund. Math. 62 (1968), 223-254.

2. - Theory of retracts, Monografie Matematyczne, Tom 44, PWN, Warsaw, 1967, $251 \mathrm{pp}$.

3. M. Brown, $A$ proof of the generalized Schoenflies theorem, Bull. Amer. Math. Soc. 66 (1960), 74-76.

4. O. Hanner, Some theorems on absolute neighborhood retracts, Ark. Mat. 1 (1951), 389-408.

5. D. R. McMillan, A criterion for cellularity in a manifold, Ann. of Math. 79 (1964), 327-337.

INSTITUTE FOR ADVANCED STUdY 